Soc Psychol Personal Sci. 2019 January ; 10(1): 121-130. doi:10.1177/1948550617746218.

\title{
The vicarious effects of discrimination: How partner experiences of discrimination affect individual health
}

\author{
Nyla Wofford ${ }^{1}$, Andrew M. Defever ${ }^{2}$, and William J. Chopik ${ }^{2}$ \\ ${ }^{1}$ Prairie View A\&M University \\ ${ }^{2}$ Michigan State University
}

\begin{abstract}
Little is known about how discriminatory experiences are associated with interpersonal relationships-specifically whether one person's experience of discrimination has psychological effects on their partner and their relationship (i.e., vicarious effects). Using dyadic data analyses, we examined actor and partner effects of discriminatory experiences on self-rated health, chronic illness, depression, and relationship strain in a sample of 1,949 couples (3,898 participants). Actor and partner discrimination were associated with poorer health, greater depression, and greater relationship strain. These effects were mediated by higher levels of relationship strain. Our findings provide insight into the effects of direct and vicarious experiences of discrimination on interpersonal relationships.
\end{abstract}

\section{Keywords}

discrimination; depression; physical health; interpersonal relationships

Individual experiences of discrimination can vary dramatically—based on race, gender, weight, sexual orientation, and other social categories-and there is a robust literature linking these experiences of discrimination with stress and poorer mental and physical health (Armstead et al., 1989; Krieger, 1990; Seeman et al., 2004; Torres \& Vallejo, 2015). Furthermore, extant research suggests that emotions and stress can "spread" through social networks (Rosenquist, Fowler, \& Christakis, 2011). Likewise, there is a large body of research on how stress experienced in one domain (e.g., work) can have negative effects on functioning in another domain (e.g., family relationships; Grzywacz, Almeida, \& McDonald, 2002). While most research focuses on the negative outcomes for those who directly experience discrimination, considerably less is known about how these stressful experiences affect the people around them. Thus, the current study examines how discrimination experienced by one person is associated with the mental and physical health of their romantic partner.

Correspondence concerning this manuscript should be addressed to William J. Chopik, Department of Psychology, Michigan State University, 316 Physics Rd., East Lansing, Michigan 48824. chopikwi@msu.edu.

Any and all materials associated with this manuscript can be accessed by request to the corresponding author. 


\section{The effects of discrimination on individuals}

Discrimination is associated with negative mental and physical health outcomes (Williams \&

Mohammed, 2009). The negative consequences of discrimination experiences likely result from several processes. These experiences are more pronounced when discrimination is experienced repeatedly, leading to increased depressive symptoms, poorer physical health, and more chronic illness, possibly through the activation of stress response systems or changes in health behavior (Adam et al., 2015; Fuller-Rowell, Doan, \& Eccles, 2012). Indeed, exposure to racial/ethnic discrimination is perceived as traumatic and stressfulleading to higher anxiety, feelings of hopeless and helplessness, and increased depressive symptoms (Armstead et al., 1989; Bullock \& Houston, 1987; Clark et al., 1999; Fernando, 1984; Torres \& Vallejo, 2015). Stressors resulting from discriminatory experiences can also cause physical depletion, premature illness, and mortality (Andersen, Kiecolt-Glaser, \& Glaser, 1994; Cacioppo, 1994; S. Cohen \& Herbert, 1996; Herd, 1991; Seeman et al., 2004). For example, experiences of racism are associated with elevated blood pressure in African Americans across the lifespan (Armstead et al., 1989; Center for Disease Control and Prevention, 2015; Krieger, 1990). These elevated blood pressure levels are thought to be one of the mechanisms for explaining racial group differences in longevity.

\section{The effects of discrimination on close others}

Research on discrimination has largely focused on an individual's direct experiences of discrimination. We draw on two literatures that would suggest possible links between one person's discrimination experiences and another person's mental and physical healthemotional contagion and stress spillover.

Emotional contagion refers to the tendency to catch, experience, or express another person's emotion by interacting with them and/or mimicking facial, vocal, and postural expressions (Hatfield, Cacioppo, \& Rapson, 1994). Large epidemiological studies have demonstrated that happiness, depression, health, and health behavior can spread through social networks (Christakis \& Fowler, 2007; Hill et al., 2010; Rosenquist et al., 2011). Emotions can also be transmitted through the tone and content of conversations, interactions, and long-term relationship dynamics (Hoppmann \& Gerstorf, 2009). For example, Totterdell and colleagues (2012) found that individuals who witness unpleasant interactions (like discrimination) happening to others report experiencing emotional drain, a symptom of emotional exhaustion (Totterdell \& Parkinson, 1999). Such emotional exhaustion could affect an person's interactions and relationships with other people, leading to negative outcomes for both the individual and the people around them. These indirect experiences of discrimination—vicarious discrimination experiences — have the potential to affect health and well-being (Settles, Minor-Rubino, \& Jellison, 2004). Further, we expect the effects of vicarious discrimination to be especially pronounced among people in intimate relationships, as they have higher degrees of emotional contagion (Christakis \& Fowler, 2007; Kimura, Daibo, \& Yogo, 2008). The stress conferred from recurrent discriminatory experiences may spread from an individual into their close relationships (via emotional contagion) and disrupt their partner's mental and physical health. 
A related literature on stress spillover-how stressors external to a close relationship increase maladaptive relationship behavior and compromise relationship satisfaction-also supports a possible link between partner discrimination experiences and individual health and well-being (Randall \& Bodenmann, 2009). The preponderance of evidence from these studies examining stress spillover suggests that stress originating from other sources (e.g., finances, work, school) has negative effects on relationship well-being primarily through decreases in emotion regulation abilities and increases in negative relationship behaviors (Brock \& Lawrence, 2008; Buck \& Neff, 2012; Neff \& Broady, 2011; Neff \& Karney, 2004, 2007). For example, in a sample of Latino newlyweds, Trail and colleagues (2012) found that individual experiences of discrimination were associated with worse marital quality and aggression for both individuals and their partners. This study suggests that one of the pathways that link discrimination experiences and well-being across people might be explained by the negative relational behavior enacted by individuals in response to external stressors.

\section{The Current Study}

We hypothesized that an individual's and their partner's discrimination experiences would be linked to worse health and well-being for individuals. Further, given the large literature on how negative relationship interactions have deleterious effects on health and well-being (House, Landis, \& Umberson, 1988; Rook, 2015; Sarason, Sarason, \& Gurung, 2001), we also hypothesized that relationship strain would mediate the links between individual/ spousal discrimination experiences and mental and physical health. Our work is distinct from previous work in that it measures health outcomes within dyads and tested relationship dynamics as a mediator linking discrimination to health and well-being. Previous work has relied primarily on individual reports of discrimination and has not explored the downstream health consequences of vicarious discrimination experiences for individuals (Torres \& Vallejo, 2015; Trail et al., 2012).

Because members of minority groups are more likely to be the target of discrimination, we also ran supplementary analyses examining the effects of discrimination experiences specifically resulting from different sources (e.g., age, race, and gender). We also examined whether the vicarious effects of discrimination were stronger for ethnic minorities, as these individuals are disproportionately targets of discrimination (Plummer \& Slane, 1996).

\section{Methods}

\section{Participants}

Participants consisted of 1,949 couples (3,898 individuals) ranging in age from 50 to 94 ( $M$ $=66.81, S D=8.92$ ). The racial composition was $83.7 \%$ Caucasian, $8.1 \%$ African-American, $6.4 \%$ Hispanic, and $1.8 \%$ other. Median education level was high school $(15.1 \%$ had less than a high school education, $36.9 \%$ had a high school education or GED, $48.0 \%$ had at least some college education). 


\section{Materials \& Procedure}

The Health and Retirement Study (HRS), sponsored by the National Institute on Aging conducted by the University of Michigan, is a nationally representative prospective panel study that has surveyed over 22,000 Americans aged 50+ since 1992 (Sonnega et al., 2014). The current sample comprises data from heterosexual couples within the HRS in which both husband and wife completed all target measures ( $N=1,949$ couples or 3,898 individuals). We used all available data for the current report and implemented no stopping rule or exclusionary criteria.

Everyday experiences of discrimination-Discrimination was assessed with five items designed to capture the hassles and stress associated with perceived discrimination (Williams et al., 1997). Participants responded with how often a series of events happened to them on a 6-point scale ranging from 1(never) to 6(almost everyday). The five items were, "You are treated with less courtesy or respect than other people," "You receive poorer service than other people at restaurants or stores," "People act as if they think you are not smart," "People act as if they are afraid of you," and "You are threatened or harassed." Responses were averaged across the five items to yield a composite of experiences of discrimination $(a=.81 ; M=1.66, S D=.71)$.

Participants could also indicate $(1=$ yes, $0=$ no) whether the discriminatory experience could be attributed to their age, race, gender, ancestral or national origin, weight, physical disability, sexual orientation, another aspect of their physical appearance, or another reason entirely. Specific reasons beyond these categories are unavailable. Because the frequency of many of these attributions was so low ( $\leq 5 \%)$, our first supplementary analysis focused on modeling the most common forms of discrimination—racial (experienced by $7.5 \%$ of the sample), gender (12.5\%), and age discrimination (28.6\%); however, the analysis still includes the full sample and their general discrimination experiences. Further, participants indicated the reason for being discriminated against for all five discrimination questions together. This is a limitation of the current study as we cannot definitively measure which exact experiences (e.g., "You are threatened or harassed.") result from certain forms of discrimination. Rather, HRS collects a summary measure of whether respondent's collective experiences of discrimination result from different sources.

Self-rated health-Subjective health was assessed on a five-point scale in response to the item, "Would you say your health is excellent, very good, good, fair, or poor?" Responses were coded in numeric order such that higher values indicate better health $(M=3.34, S D=$ $1.04)$.

Chronic disease-Each participant reported whether they have ever suffered from high blood pressure, diabetes, cancer or a malignant tumor of any kind, lung disease, coronary heart disease including heart attacks and congestive heart failure, emotional, nervous, or psychiatric problems, arthritis or rheumatism, and stroke. The number of major health problems was summed such that higher values indicate a greater presence of chronic disease $(M=1.90, S D=1.36)$. 
Depression-Depression was assessed using a modified eight-item version of the Center for Epidemiological Studies Depression Scale (CES-D; Radloff, 1977). Participants indicated whether or not they experienced any of these symptoms in the past week: feeling depressed, feeling everything they did was an effort, restless sleep, happiness (reverse coded), loneliness, enjoying life (reverse coded), sad, and feeling unmotivated. The number of depressive symptoms was summed, with higher values indicating higher levels of depression $(M=1.08, S D=1.65)$. Internal consistency for the CES-D was high, $a=.82$ (Ayotte, Yang, \& Jones, 2010).

Relationship strain-Relationship strain was measured with four questions about an individual's spousal relationship (Chopik, 2017; Uchino, 2009). These items are (1) "How often do they make too many demands on you?" (2) "How much do they criticize you?" (3) "How much do they let you down when you are counting on them?" and (4) "How much do they get on your nerves?" Participants responded on a scale ranging from 1(not at all) to 4(a $(o t)$. Responses were averaged across the four items to yield an overall composite of relationship strain $(a=.78 ; M=1.97, S D=.65)$.

Relationship closeness-Relationship closeness was measured with a single item, "How close is your relationship with your spouse or partner?" Participants responded on a scale ranging from 1 (not at all close) to 4 (very close).

\section{Statistical Approach}

Multi-level modeling (MLM) procedures recommended for dyadic data analysis were used (Kenny, Kashy, \& Cook, 2006). MLM estimates both actor effects (associations between a person's discrimination and his/her own health) and partner effects (associations between a person's discrimination and his/her partner's health) while accounting for the statistical nonindependence of members in a couple. Both actor and partner effects of discrimination were tested as predictors of chronic illness, self-rated health, depression, and relationship strain. Statistical analyses were conducted using the SPSS MIXED procedure (Peugh \& Enders, 2005).

Following recommended procedures (Kenny et al., 2006), gender was contrast-coded $(-1=$ men, $1=$ women). Chronic illness, self-rated health, depression, and relationship strain served as the dependent measures. Separate multi-level models were conducted predicting each of the physical/mental health measures from actor discrimination and partner discrimination. Individual-level covariates (age, gender, education, and relationship closeness) were also included in each model. The covariates were included to further isolate the effect of discrimination on mental and physical health by partialling out variance in each outcome attributable to these sources. Importantly, the results reported below are the same with and without each covariate included in the models. We thus retained and reported the models with the covariates included. Interactions between actor and partner discrimination were tested for the possibility of a multiplicative effect on physical/mental health.

Mediation analyses were conducted using structural equation modeling with nested models comparing fit between full and partial mediation models. Relationship strain was modeled separately for both couple members as mediators of the link between actor/partner 
discrimination and mental and physical health. This mediation model tests whether one partner's experiences of discrimination are associated with higher levels of marital strain in the other partner (Trail et al., 2012) and subsequently whether higher levels of marital strain are associated with worse mental and physical health (Chopik, 2017; Rook, 2015).

\section{Results}

\section{Preliminary Results}

Descriptive statistics and preliminary correlations are presented in Table 1 for men (lower diagonal) and women (upper diagonal). Discrimination experiences were associated with poorer self-rated health, greater depression, and greater relationship strain. Having a partner who has been discriminated against was associated with poorer self-rated health (for men only), greater depression, and greater relationship strain. Discrimination experiences were unrelated to chronic illnesses. Actor and partner discrimination experiences were correlated within couples, such that if one person experienced discrimination, their partner was also likely to have experienced discrimination.

\section{Multi-level Results}

Multi-level models examining actor and partner discrimination experiences predicting mental and physical health are presented in Tables 2 (chronic illness), 3 (self-rated health), 4 (depression), and 5 (relationship strain). Actor discrimination was associated with more chronic illnesses, poorer self-rated health, greater depression, and greater relationship strain. Partner discrimination was associated with poorer self-rated health, greater depression, and greater relationship strain. There was no evidence for any actor discrimination $\times$ partner discrimination effects on physical or mental health (all $p \mathrm{~s}>.25$ ). There was a significant actor discrimination $\times$ partner discrimination effect for marital strain $(b=-.03, p=.008, r=$ -.07). This interaction can be seen in Figure 1.

Simple slopes analyses revealed that the effect of partner discrimination on relationship strain was stronger among actors who experienced little $(-1 \mathrm{SD})$ discrimination themselves $(b=.15, p<.001)$ compared to actors who experienced more $(+1 \mathrm{SD})$ discrimination $(b=$. $10, p<.001)$. This suggests that the effects of partner discrimination are associated with more relationship strain among individuals who have less experience with discrimination themselves. Although we are speculating, it could be that individuals who are rarely discriminated against may find their partner's experiences with discrimination more alarming and upsetting given the lack of their own discriminatory experiences from which to judge. Likewise, the effect of partner discrimination could be less influential among those who experience a lot of discrimination because their commiseration with similar experiences (which still affects their health and well-being) is less likely to translate to relationship strain. ${ }^{1}$

\footnotetext{
${ }^{1}$ Because of the panel nature of HRS, many of these outcomes (chronic illnesses, self-rated health, and depression) are available at multiple waves, spaced two years apart. To test the enduring effects of actor/partner discrimination on health, we re-ran our main models but predicting 2008 outcomes while controlling for individual's standing on these outcomes in 2006. Actor discrimination predicted worse health over time (chronic illnesses: $b=.03, p=.01$; self-rated health: $b=-.06, p=.003$; depression: $b=.16, p<$. 001). Partner discrimination did not predict worse health over time (chronic illnesses: $b=-.01, p=.48$; self-rated health: $b=-.03, p$ $=.06$; depression: $b=-.003, p=.92$ ). The enduring effect of experiences with discrimination predicting worse health conceptually
} 
None of the effects reported above were distinguishable (i.e., moderated) by gender, such that the effects of actor/partner discrimination on mental, physical, and relational health were similar for men and women.

\section{Are the negative effects of discrimination specific to the type of discrimination?}

In a series of follow-up analyses, we examined how particular forms of discrimination (e.g., race, gender, age) were associated physical and mental health. These analyses test whether the presence of discrimination is more harmful if the discrimination is attributable to an actor/partner's age, gender, or race. Forms of discrimination (discrimination due to race, gender, and age for both actors and partners) were entered into the aforementioned models as predictors of chronic illness, self-rated health, depression, and relationship strain. Both main effects (i.e., being the subject of racial discrimination overall) and interactions with discrimination frequency (i.e., the frequency of racial discrimination) were entered into the models. The full multi-level models can be found in Supplementary Tables 1-4.

For the most part, specific attributions of discrimination were unrelated to mental and physical health, with two exceptions. There was a significant actor gender discrimination $\times$ frequency interaction for both chronic illnesses $(b=-.23, p=.012, r=-.05)$ and self-rated health $(b=.16, p=.025, r=.04)$. Among those experiencing some form of gender discrimination, the frequency of that discrimination was not a significant predictor of chronic illnesses $(b=.08, p=.544, r=.03)$ or self-rated health $(b=.04, p=.712, r=.02)$. This suggests that experiencing even small and moderate amounts of gender discrimination can be as toxic to health as experiencing large amounts of gender discrimination. Among those experiencing discrimination that was not attributable to gender (but possibly other forms), frequency of discrimination was a significant predictor of both chronic illnesses $(b$ $=.15, p<.001, r=.06)$ and self-rated health $(b=-.13, p<.001, r=-.07$; see Supplementary Figure 1). Aside from these two interactions, the type of discrimination experienced by actors and partners is largely unrelated to physical health, mental health, and relationship strain. Discrimination was associated with negative outcomes, regardless of the type of discrimination. ${ }^{2}$

\section{Are the negative effects of actor and partner discrimination stronger for ethnic minorities?}

In another series of follow-up analyses, we examined whether the effects of actor and partner discrimination were stronger among ethnic minorities. Because the majority of the

replicates past longitudinal research. The question for why partner discrimination does not predict health longitudinally is less clear. It could be the case that others' experiences have more of a fleeting influence on our health and well-being relative to our own experiences (Campbell et al., 2014; O'Brien \& Kardas, 2016). Likewise, the effect of a partner's experiences on individuals may take many years to accumulate, and we only capture some of this accumulation cross-sectionally. Or there could be more complex ways in which partner discrimination experiences and individual mental/physical health change in concert over time (Torres \& Ong, 2010). Unfortunately, HRS does not have parallel measurements of discrimination and health to enable the modeling of such processes. Nevertheless, we felt it necessary to disclose that additional measurements of health were available and that the effect of partner discrimination did not persist over time (Simmons, Nelson, \& Simonsohn, 2011; Vazire, 2015). Future research can further examine the conditions and time course under which partners' experiences affect individual health and well-being.

${ }^{2}$ We also tested whether any of the effects of racial, gender, or age discrimination differed between men and women. The vast majority of the effects were consistent in size and significance between men and women (i.e., were not moderated by gender). The few that were moderated by gender were inconsistent across outcomes, demonstrated p-values just below .05, were not significant for either men or women when examined within each group, and were generally confusing or uninterpretable given the low incidence of each type of discrimination. Thus, we hesitate to devote too much space to these interactions, especially given the high family-wise error of running so many follow-up tests. However, we have made them and their decompositions available in the Supplementary Materials. 
sample was Caucasian (83.7\%) and each minority group comprised less than $10 \%$ of the sample, we recoded the race variable for this particular analysis such that $0=$ Caucasian and $1=$ Ethnic Minority. Actor minority status and its interaction with actor discrimination frequency and partner discrimination frequency were entered into the aforementioned multilevel models. These analyses test whether discrimination frequency is more strongly related to each outcome among ethnic minority participants compared to Caucasian participants. The results from these models can be found in Supplementary Tables 5-8. Consistent with the previous models, ethnic minority participants reported worse self-rated health, more depression, and more relationship strain than Caucasian participants (Is > .04). However, there were no significant actor/partner discrimination $\times$ minority status interactions $(p s>$. 082), suggesting that the effects of perceived discrimination for both actors and partners were equally harmful among Caucasian and ethnic minority participants. ${ }^{3}$

\section{Does relationship strain mediate the effects of actor/partner discrimination on mental and physical health?}

We also tested a dyadic mediation model in which actor/partner discrimination experiences predicted both actor/partner relationship strain which in turn predicted mental and physical health for individuals (see Supplementary Figures 2-4 for full models and estimates). We only modeled an individual's relationship strain on individual outcomes. Central to this decision was the way in which strain was measured. Instead of a typical assessment of strain (e.g., my relationship is strenuous or I am unhappy in my relationship), the HRS assessment of strain focuses on the behavior of one's partner (e.g., how much does your partner criticize you, how much does your partner get on your nerves). We expected the actor effect to be larger in size (i.e., that an individual getting on their partner's nerves primarily affects their partner's health and well-being) compared to the partner effect (i.e., that an individual getting on their partner's nerves (reported by their partner) primarily affects their own health and well-being; a "partner regard" effect). However, in modeling this cross-partner 'b" path (in which an individual's felt strain from their partner affects their partner's health and wellbeing), we found there were no significant effects of this "partner regard" effect (Murray, Holmes, \& Griffin, 1996). Thus, this path was not included in the final model depicted in Supplementary Figures 2-4.

We used the bootstrapping procedure recommended by MacKinnon (2008) and nested models to test for full and partial mediation (Manne et al., 2015; Manne et al., 2014). The fully mediated model showed good fit across different indices for chronic illnesses $\left(\chi^{2}(6)=\right.$ 4.53, $p<.001$. CFI $=.977$, RMSEA $=.043)$, self-rated health $\left(\chi^{2}(6)=8.98, p<.001\right.$. CFI $=.951$, RMSEA $=.016)$, and depression $\left(\chi^{2}(6)=22.03, p<.001 . \mathrm{CFI}=.888\right.$, RMSEA $=$. 037). An additional model specifying partial mediation was also included, in which direct paths from actor/partner discrimination to actor/partner mental and physical health were specified. In these models, however, model fit either did not change or became slightly worse ( $\triangle$ RMSEAs ranged from .007 to .045). This suggests that the actor and partner effects

\footnotetext{
${ }^{3} \mathrm{We}$ also tested whether the effects of actor/partner discrimination on all of our outcomes among minorities differed between men and women. Nearly all of the effects were consistent in size and significance between men and women (i.e., were not moderated by gender). The one exception was a finding in which actor discrimination frequency predicted depression among white men only $(b=$. 22, $p=.028)$. This interaction is formally decomposed in the Supplementary Materials.
} 
of discrimination on mental and physical health were fully mediated by the inclusion of relationship strain. Path estimates were consistent with those reported from the multi-level analyses reported above. Direct effects of actor/partner discrimination on each of the outcomes was reduced in size, often to non-significance, when including the indirect effect through relationship strain, further suggesting mediation.

In a series of supplementary analyses, we examined whether the mediation process differed between men and women by comparing a model in which paths between men and women were constrained to be equal to a model in which they were freely estimated. The nested model tests suggested that the majority of the paths were the same for men and women, with the exception of one ' $b$ ' path from strain to chronic illness for women. The direction and interpretation of the mediational process is the same as the other models, however, this ' $b$ ' path from the mediator (strain) to the outcome (chronic illnesses) did not reach statistical significance for women (see Supplementary Figure 2 for an expanded discussion).

\section{Discussion}

In the current study, we examined how one person's experiences of discrimination were associated their own mental and physical health and the mental and physical health of their partner. We found an association between actor and partner experiences of discrimination and many of our outcomes-more frequent experiences of discrimination for both actors and partners were associated with poorer self-rated health, higher depression, and more relationship strain. This is consistent with previous research examining associations between experiences of discrimination and poor mental and physical health at the individual level. These effects are also consistent with work demonstrating the deleterious effects that discrimination has on relational processes (Trail et al., 2012). Further, many of the links between actor/partner discrimination and mental and physical health were mediated by higher levels of relationship strain.

We found that direct experiences of discrimination were associated with poorer mental and physical health. This effect may be due in part to the stress and negative emotions that accompany discrimination and their downstream effects on close relationships (Fernando, 1984; Torres \& Vallejo, 2015; Trail et al., 2012). We found additional support for the notion that discrimination experiences are linked to health through stress and compromised social relationships. Actor discrimination experiences were associated with higher levels of relationship strain, which were in turn associated with poorer health and well-being. We also found a vicarious discrimination effect, such that having a partner who experienced discrimination was associated with more depression and poorer health for individuals (Hatfield et al., 1994). This partner discrimination-health path was also mediated by higher levels of relationship strain. The dyadic effects of discrimination on relationship strain are also consistent with previous research (Trail et al., 2012). In the current study, we extended this effect by further linking the discrimination-relationship strain link to mental and physical health in a sample of older couples. Although we tested the mediating effect of relationship strain on our mental and physical health outcomes, future research should examine more proximal mediators of these vicarious effects, such as physiological stress indices (Armstead et al., 1989; Bullock \& Houston, 1987; Clark et al., 1999). 
The finding that one person's experiences of discrimination were associated with mental, physical, and relational health outcomes in their partner aligns well with existing research examining similar cross-over effects within couples and emotional contagion more broadly (Neff \& Karney, 2007; Rosenquist et al., 2011; Trail et al., 2012). Like many of these studies, our study did not have the fidelity to examine exactly how the stress resulting from these experiences crosses over to affect romantic partners. In some cases, partners might explicitly disclose experiences of discrimination with each other, as they do with other details in their lives (Greene, Derlega, \& Mathews, 2006). This disclosure of negative experiences might increase stress in the partner who receives this disclosure given that partners consider each other as an extension of themselves (Aron \& Aron, 1996). In other cases, partners might not disclose a discrimination experience but the stress from this experience might spillover and affect their behavior toward their partner. Experiences of discrimination often invoke feelings of anger and externalizing responses (e.g., yelling, throwing things, fighting), making it more likely that relationship interactions are tinged with additional hostility (Caldwell et al., 2004; Hansen \& Sassenberg, 2006; Scott \& House, 2005; Trail et al., 2012). An important direction for future research is examining how the ways in which a partner discloses discrimination experiences can decrease the likelihood that this stress translates to increased relationship strain and negative health outcomes. To our knowledge, the benefits (or drawbacks) of partner disclosures of discrimination experiences have not been the subject of empirical study.

There were some limitations to the current study. As minority groups are—by definitionless represented in society, it was difficult to get a large sample with sufficient power to test more specific associations of particular types of discrimination. For instance, there were very few interracial couples present in the sample, preventing us from analyzing the vicarious effects of discrimination within these couples. Another limitation is that HRS did not assess coping responses, which could serve as a moderators or mediators of the effects observed in the current study. It has been found that coping strategies often differ by race and can influence reports of re-experiencing discrimination (Sanders Thompson, 2006). Likewise, there are probably additional mechanisms that can explain the link between discrimination, relationship strain, and mental/physical health that were not measured by the HRS (e.g., aggressive behavior, negative partner cognitions, self-esteem changes).

Many of the effects in the current study were small in magnitude. Although we had a large number of couples, large sample sizes may yield findings that are statistically significant but of little practical significance (J. Cohen, 1990). Therefore, findings should be interpreted in light of how these constructs operate in the real world and discrimination affects couples' health and well-being among a broad sample of older couples (Abelson, 1985).

\section{Conclusion}

The purpose of this study was to explore the existence of actor and partner effects of discrimination in effort to catalyze future study in how stressful experiences are associated with the health of couples. Research in this area has narrowly focused on the individual's direct experiences of discrimination without taking into account in the shared context of close relationships. However, the current study provides the groundwork for further 
exploration of how stressful experiences spread through social networks and influence mental and physical health.

\section{Supplementary Material}

Refer to Web version on PubMed Central for supplementary material.

\section{Acknowledgments}

Research reported in this publication was supported by the National Institute of General Medical Sciences of the National Institute of Health under Award Number 1R03AG054705-01A1. The content is solely the responsibility of the authors and does not necessarily represent the official views of the National Institutes of Health. The Health and Retirement Study is sponsored by the National Institute on Aging (NIA U01AG09740) and is conducted by the University of Michigan.

The authors would like to acknowledge Jeewon Oh, David Johnson, and members of the Close Relationships Lab for their comments on previous versions of the manuscript.

\section{References}

Abelson RP. 1985; A variance explanation paradox: When a little is a lot. Psychological Bulletin. 97(1):129-133.

Adam EK, Heissel JA, Zeiders KH, Richeson JA, Ross EC, Ehrlich KB, Eccles JS. 2015; Developmental histories of perceived racial discrimination and diurnal cortisol profiles in adulthood: A 20-year prospective study. Psychoneuroendocrinology. 62:279-291. [PubMed: 26352481]

Andersen BL, Kiecolt-Glaser JK, Glaser R. 1994; A biobehavioral model of cancer stress and disease course. American psychologist. 49(5):389. [PubMed: 8024167]

Armstead CA, Lawler KA, Gorden G, Cross J, Gibbons J. 1989; Relationship of racial stressors to blood pressure responses and anger expression in black college students. Health Psychology. 8(5): 541. [PubMed: 2630293]

Aron, A, Aron, EN. Self and self expansion in relationships. In: Fletcher, GJO, Fitness, J, editorsKnowledge structures in close relationships: A social psychological approach. Mahway, NJ: Lawrence Erlbaum Associates; 1996. 325-344.

Ayotte BJ, Yang FM, Jones RN. 2010; Physical health and depression: A dyadic study of chronic health conditions and depressive symptomatology in older adult couples. The Journals of Gerontology Series B: Psychological Sciences and Social Sciences. 65B(4):438-448.

Brock RL, Lawrence E. 2008; A longitudinal investigation of stress spillover in marriage: Does spousal support adequacy buffer the effects? Journal of Family Psychology. 22(1):11. [PubMed: 18266528]

Buck AA, Neff LA. 2012; Stress spillover in early marriage: The role of self-regulatory depletion. Journal of Family Psychology. 26(5):698. [PubMed: 22866931]

Bullock SC, Houston E. 1987; Perceptions of racism by Black medical students attending White medical schools. Journal of the National Medical Association. 79(6):601-608. [PubMed: 3612829]

Cacioppo JT. 1994; Social neuroscience: Autonomic, neuroendocrine, and immune responses to stress. Psychophysiology. 31(2):113-128. [PubMed: 8153248]

Caldwell CH, Kohn-Wood LP, Schmeelk-Cone KH, Chavous TM, Zimmerman MA. 2004; Racial discrimination and racial identity as risk or protective factors for violent behaviors in African American young adults. American Journal of Community Psychology. 33(1):91-105. [PubMed: 15055757]

Campbell T, O’Brien E, Van Boven L, Schwarz N, Ubel P. 2014; Too much experience: a desensitization bias in emotional perspective taking. Journal of Personality and Social Psychology. 106(2):272-285. [PubMed: 24467422] 
Center for Disease Control and Prevention. High blood pressure facts. 2015. Retrieved from http:// www.cdc.gov/bloodpressure/facts.htm

Chopik WJ. 2017Associations among relational values, support, health, and well-being across the adult lifespan. Personal Relationships.

Christakis NA, Fowler JH. 2007; The spread of obesity in a large social network over 32 years. The New England Journal of Medicine. 357(4):370-379. [PubMed: 17652652]

Clark R, Anderson NB, Clark VR, Williams DR. 1999; Racism as a stressor for African Americans: A biopsychosocial model. American psychologist. 54(10):805. [PubMed: 10540593]

Cohen J. 1990; Things I have learned (so far). American Psychologist. 45(12):1304-1312.

Cohen S, Herbert TB. 1996; Health psychology: Psychological factors and physical disease from the perspective of human psychoneuroimmunology. Annual review of psychology. 47(1):113-142.

Fernando S. 1984; Racism as a cause of depression. International Journal of Social Psychiatry. 30(12):41-49. [PubMed: 6706494]

Fuller-Rowell TE, Doan SN, Eccles JS. 2012; Differential effects of perceived discrimination on the diurnal cortisol rhythm of African Americans and Whites. Psychoneuroendocrinology. 37(1):107118. [PubMed: 21689889]

Greene K, Derlega VJ, Mathews A. 2006Self-disclosure in personal relationships. The Cambridge handbook of personal relationships. :409-427.

Grzywacz JG, Almeida DM, McDonald DA. 2002; Work-family spillover and daily reports of work and family stress in the adult labor force. Family relations. 51(1):28-36.

Hansen N, Sassenberg K. 2006; Does social identification harm or serve as a buffer? The impact of social identification on anger after experiencing social discrimination. Personality and Social Psychology Bulletin. 32(7):983-996. [PubMed: 16738030]

Hatfield E, Cacioppo JT, Rapson RL. 1994; Emotional contagion. Current directions in psychological science. 2(3):96-99.

Herd JA. 1991; Cardiovascular responses to stress. Physiological Reviews. 71:305-330. [PubMed: 1986391]

Hill AL, Rand DG, Nowak MA, Christakis NA. 2010Emotions as infectious diseases in a large social network: the SISa model. Proceedings of the Royal Society B: Biological Sciences.

Hoppmann CA, Gerstorf D. 2009; Spousal interrelations in old age-A mini-review. Gerontology. 55(4):449-459. [PubMed: 19346740]

House J, Landis K, Umberson D. 1988; Social relationships and health. Science. 241(4865):540-545. [PubMed: 3399889]

Kenny, DA, Kashy, DA, Cook, WL. Dyadic data analysis. New York: Guilford Press; 2006.

Kimura M, Daibo I, Yogo M. 2008; The study of emotional contagion from the perspective of interpersonal relationships. Social Behavior and Personality: an international journal. 36(1):27-42

Krieger N. 1990; Racial and gender discrimination: risk factors for high blood pressure? Social science \& medicine. 30(12):1273-1281. [PubMed: 2367873]

MacKinnon, D. Introduction to statistical mediation analyses. Mahwah, NJ: Erlbaum; 2008.

Manne SL, Siegel S, Heckman CJ, Kashy DA. 2015Psychological distress as a mediator of the association between disease severity and occupational functioning among employed spouses of women recently diagnosed with breast cancer. Psycho-Oncology.

Manne SL, Siegel S, Kashy D, Heckman CJ. 2014; Cancer-specific relationship awareness, relationship communication, and intimacy among couples coping with early-stage breast cancer. Journal of Social and Personal Relationships. 31(3):314-334. [PubMed: 25242854]

Murray SL, Holmes JG, Griffin DW. 1996; The benefits of positive illusions: Idealization and the construction of satisfaction in close relationships. Journal of Personality and Social Psychology. 70(1):79-98.

Neff LA, Broady EF. 2011; Stress resilience in early marriage: Can practice make perfect? Journal of personality and social psychology. 101(5):1050. [PubMed: 21688919]

Neff LA, Karney BR. 2004; How does context affect intimate relationships? Linking external stress and cognitive processes within marriage. Personality and Social Psychology Bulletin. 30(2):134148. [PubMed: 15030629] 
Neff LA, Karney BR. 2007; Stress crossover in newlywed marriage: A longitudinal and dyadic perspective. Journal of Marriage and Family. 69(3):594-607.

O'Brien E, Kardas M. 2016; The implicit meaning of (my) change. Journal of Personality and Social Psychology. 111(6):882-894. [PubMed: 27537272]

Peugh JL, Enders CK. 2005; Using the SPSS Mixed Procedure to Fit Cross-Sectional and Longitudinal Multilevel Models. Educational and Psychological Measurement. 65(5):717-741.

Plummer DL, Slane S. 1996; Patterns of coping in racially stressful situations. Journal of Black Psychology. 22(3):302-315.

Radloff LS. 1977; The CES-D Scale: A self-report depression scale for research in the general population. Applied Psychological Measurement. 1(3):385-401.

Randall AK, Bodenmann G. 2009; The role of stress on close relationships and marital satisfaction. Clinical psychology review. 29(2):105-115. [PubMed: 19167139]

Rook KS. 2015; Social networks in later life: Weighing positive and negative effects on health and well-being. Current Directions in Psychological Science. 24(1):45-51. [PubMed: 26366047]

Rosenquist JN, Fowler JH, Christakis NA. 2011; Social network determinants of depression. Molecular Psychiatry. 16(3):273-281. [PubMed: 20231839]

Sanders Thompson VL. 2006; Coping responses and the experience of discrimination. Journal of Applied Social Psychology. 36(5):1198-1214.

Sarason, BR, Sarason, IG, Gurung, RAR. Close personal relationships and health outcomes: A key to the role of social support. In: Sarason, BR, Duck, S, editorsPersonal relationships: Implications for clinical and community psychology. New York, NY US: John Wiley \& Sons Ltd; 2001. 15-41.

Scott LD, House LE. 2005; Relationship of distress and perceived control to coping with perceived racial discrimination among black youth. Journal of Black Psychology. 31(3):254-272.

Seeman TE, Crimmins E, Huang MH, Singer B, Bucur A, Gruenewald T, Reuben DB. 2004; Cumulative biological risk and socio-economic differences in mortality: MacArthur studies of successful aging. Social science \& medicine. 58(10):1985-1997. [PubMed: 15020014]

Settles, IH, Minor-Rubino, KN, Jellison, WA. Paper presented at the Meeting for the American Psychological Association. 2004. Psychological and academic effects of direct and vicarious discrimination.

Simmons JP, Nelson LD, Simonsohn U. 2011; False-positive psychology: Undisclosed flexibility in data collection and analysis allows presenting anything as significant. Psychological Science. 22(11):1359-1366. [PubMed: 22006061]

Sonnega A, Faul JD, Ofstedal MB, Langa KM, Phillips JWR, Weir DR. 2014; Cohort profile: The Health and Retirement Study (HRS). International Journal of Epidemiology. 43(2):576-585. [PubMed: 24671021]

Torres L, Ong AD. 2010; A daily diary investigation of latino ethnic identity, discrimination, and depression. Cultural Diversity and Ethnic Minority Psychology. 16(4):561-568. [PubMed: 21058819]

Torres L, Vallejo LG. 2015; Ethnic discrimination and Latino depression: The mediating role of traumatic stress symptoms and alcohol use. Cultural Diversity and Ethnic Minority Psychology. 21(4):517. [PubMed: 25602468]

Totterdell P, Hershcovis MS, Niven K, Reich TC, Stride C. 2012; Can employees be emotionally drained by witnessing unpleasant interactions between coworkers? A diary study of induced emotion regulation. Work \& Stress. 26(2):112-129.

Totterdell P, Parkinson B. 1999; Use and effectiveness of self-regulation strategies for improving mood in a group of trainee teachers. Journal of occupational health psychology. 4(3):219. [PubMed: 10431282]

Trail TE, Goff PA, Bradbury TN, Karney BR. 2012; The costs of racism for marriage: How racial discrimination hurts, and ethnic identity protects, newlywed marriages among Latinos. Personality and Social Psychology Bulletin. 38(4):454-465. [PubMed: 22109252]

Uchino BN. 2009; Understanding the links between social support and physical health: A life-span perspective with emphasis on the separability of perceived and received support. Perspectives on Psychological Science. 4(3):236-255. [PubMed: 26158961] 
Vazire, S. Most damning result. 2015. Retrieved from http://sometimesimwrong.typepad.com/wrong/ 2015/12/most-damning-result.html

Williams DR, Mohammed SA. 2009; Discrimination and racial disparities in health: evidence and needed research. Journal of behavioral medicine. 32(1):20-47. [PubMed: 19030981]

Williams DR, Yu Y, Jackson JS, Anderson NB. 1997; Racial differences in physical and mental health socio-economic status, stress and discrimination. Journal of health psychology. 2(3):335-351. [PubMed: 22013026] 


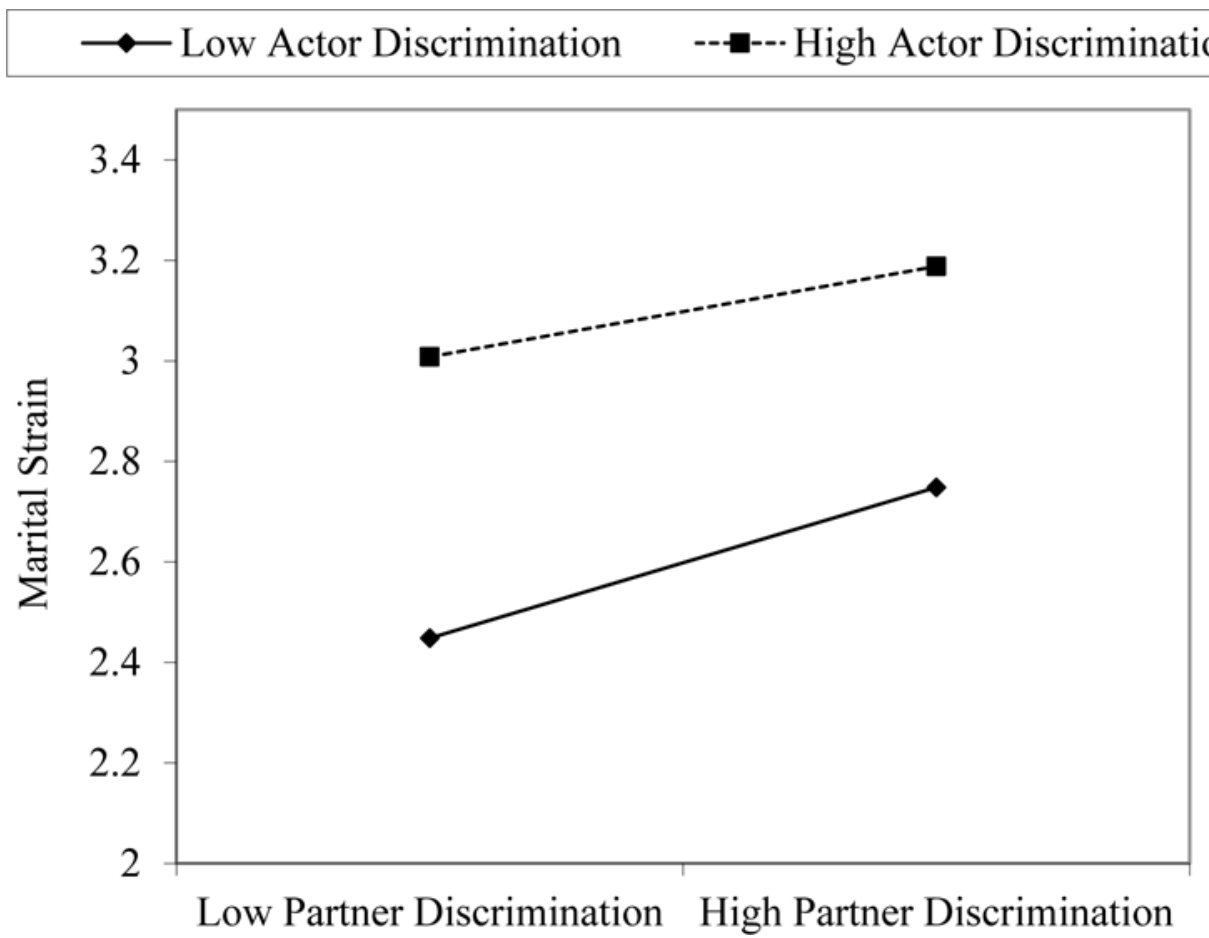

Figure 1.

Actor and partner discrimination predicting marital strain. Regression lines are plotted at one standard deviation above and below the means of actor discrimination. 
?

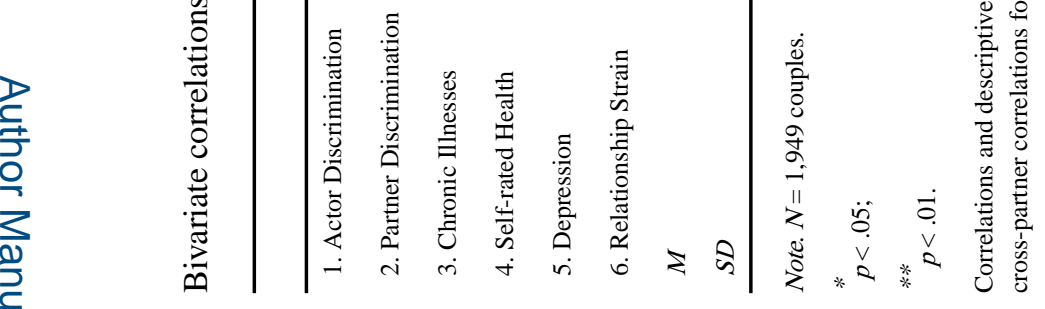

Soc Psychol Personal Sci. Author manuscript; available in PMC 2019 January 09. 


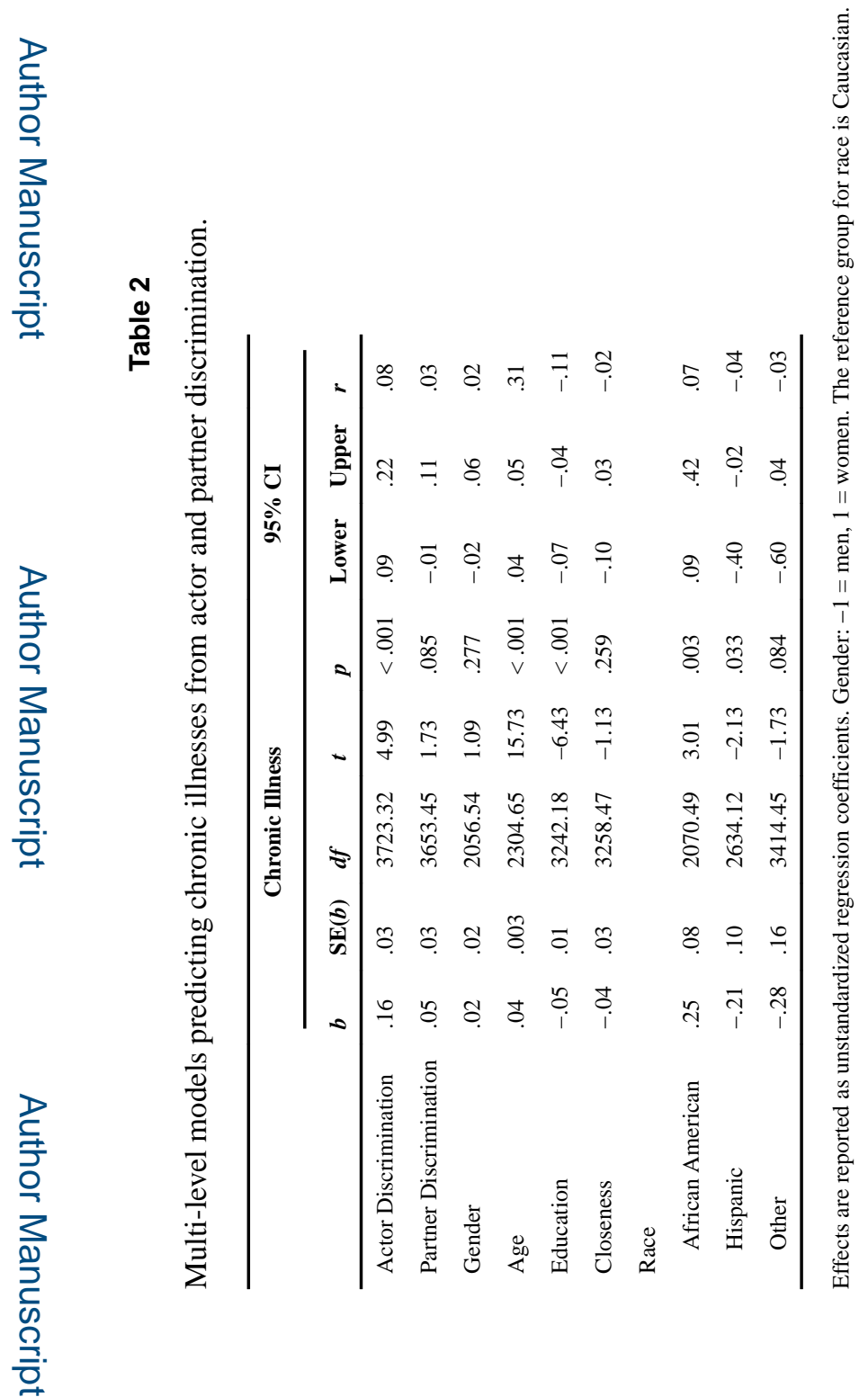




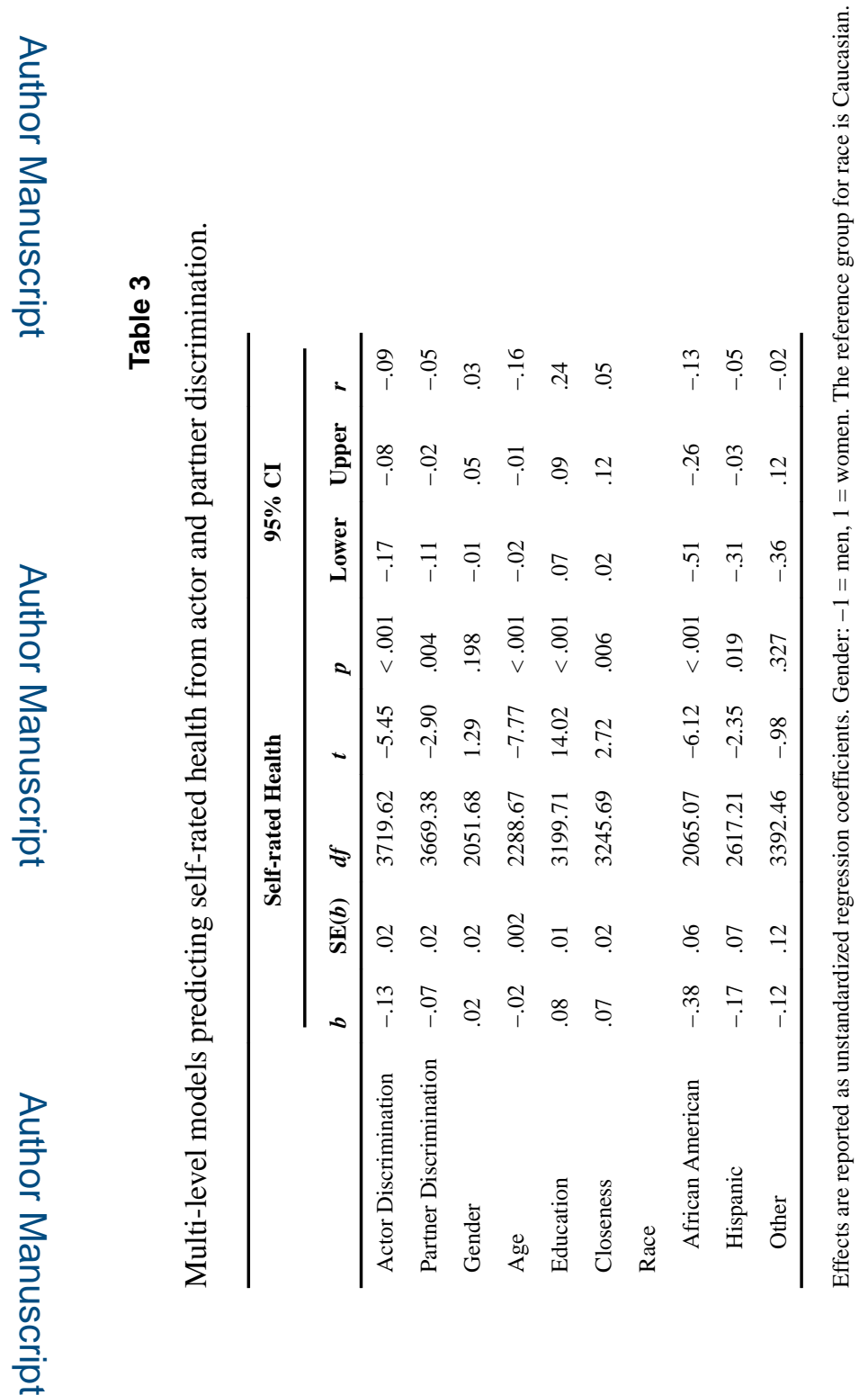




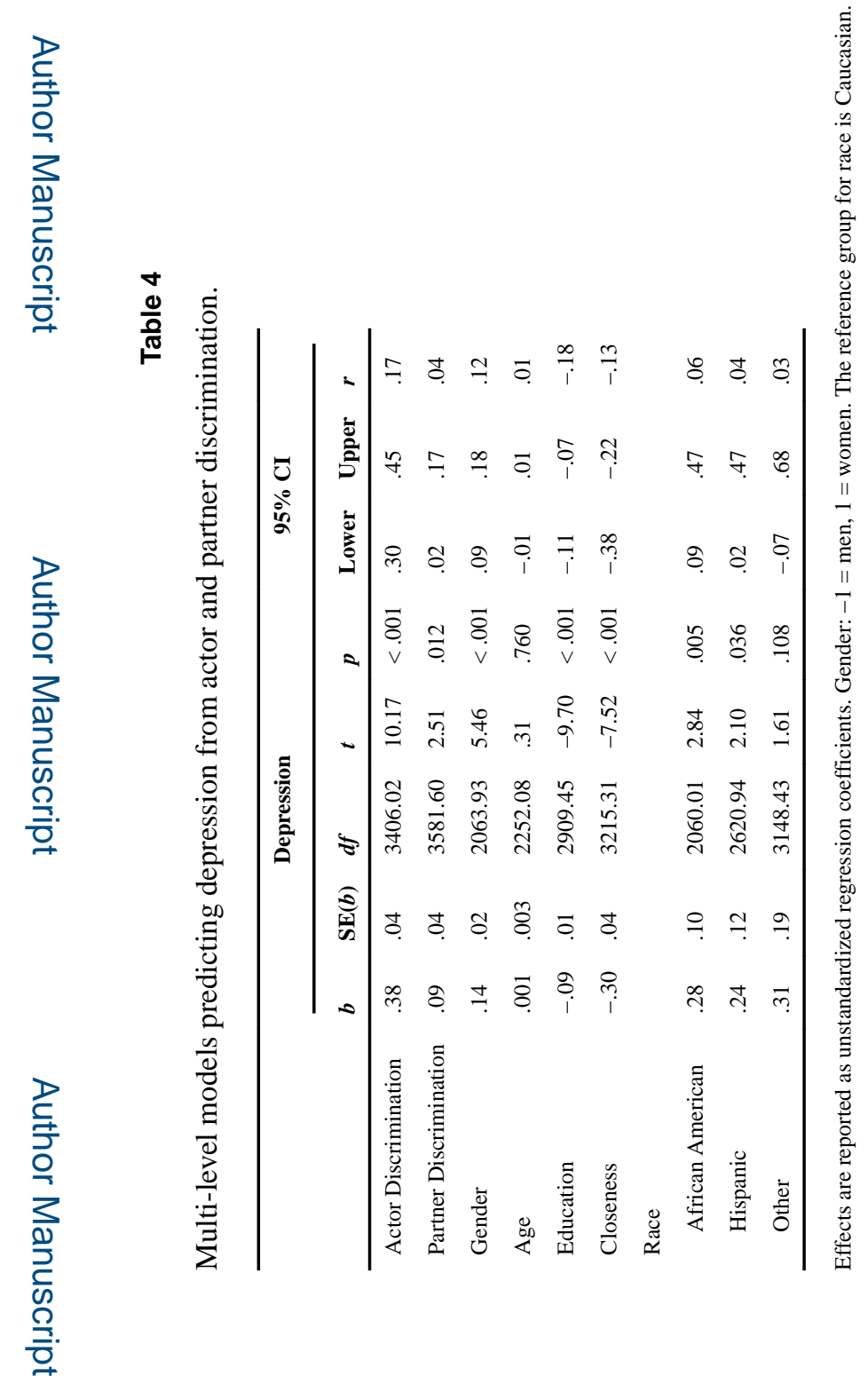




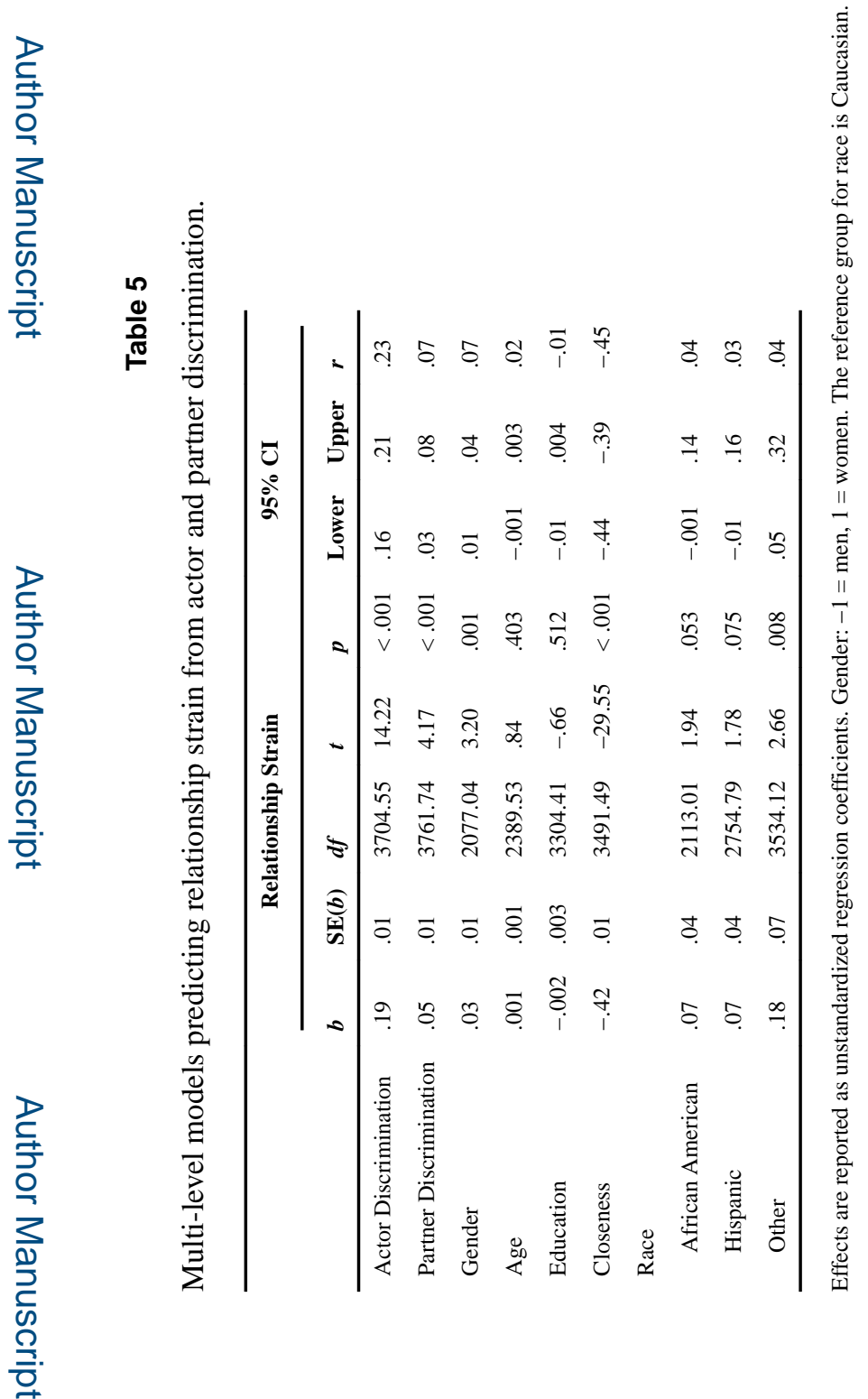

